\title{
Analyzing the Difference of Cluster, Grid, Utility \& Cloud Computing
}

\author{
Manju Sharma ${ }^{1}$, Sadia Husain ${ }^{2}$ \\ (Department of Computer Science \& Information Systems, Jazan University, Jazan, KSA)
}

\begin{abstract}
Virtualization and cloud computing is creating a fundamental change in computer architecture, software and tools development, in the way we store, distribute and consume information. In the recent era of autonomic computing it comes the importance and need of basic concepts of having and sharing various hardware and software and other resources \& applications that can manage themself with high level of human guidance. Virtualization or Autonomic computing is not a new to the world, but it developed rapidly with Cloud computing. In this paper there give an overview of various types of computing. There will be discussion on Cluster, Grid computing, Utility \& Cloud Computing. Analysis architecture, differences between them, characteristics, its working, advantages and disadvantages.
\end{abstract}

Keywords: Cluster computing, Grid computing, Utility computing, Cloud computing, Virtual machine monitor $(V M M)$.

\section{Introduction}

Grid computing is the collection of computer resources from multiple locations to achieve common goal. The grid can be thought of as a distributed system with non-interactive workloads that involve a large number of files. Grid computing is distinguished from conventional high performance computing systems such as cluster computing in that grid computers have each node set to perform a different task/application. Grid computers also tend to be more heterogeneous and geographically dispersed (thus not physically coupled) than cluster computer [Wiki].

Cloud computing $[1,4,5]$ is a computing style in which flexible and scalable IT functionalities are delivered as a service to end users using Internet. Grid computing [7] has proven to be an important field focusing on the sharing of resources and provides solution to performance as well as capacity problems for several applications.Cluster Computing [6] mainly addresses the latest results in different fields that support High Performance Distributed Computing (HPDC). Using virtualization software (VMware), it became possible to execute one or many operating systems simultaneously in an isolated environment. In section I we will discuss Cloud Computing followed by II CLOUD COMPUTING CHARACTERISTICS, III Cloud Computing Concepts and Benefits, IV Cloud computing building blocks, V. Virtual machine monitor (VMM), VI Cluster Computing, VII Cluster computing characteristics, VII Grid Computing, IX difference in Architecture of Cloud and Grid computing and X Utility Computing.

\section{Cloud Computing}

"Cloud computing is a model for enabling convenient, on-demand network access to a shared pool of configurable computing resources (e.g., networks, servers, storage, applications, and services) that can be rapidly provisioned and released with minimal management effort or service provider interaction." US National Institute of Standards and Technology, Information Technology Laboratory

Cloud computing is a comprehensive solution that delivers IT as a service. It is an Internet-based computing solution where shared resources are provided like electricity distributed on the electrical grid. Computers in the cloud are configured to work together as a unit and the various applications use the collective computing power as if they are running on a single system. The flexibility of cloud computing is a function of the allocation of resources on demand. This facilitates the use of the system's cumulative resources, negating the need to assign specific hardware to a task. Before cloud computing, websites and server-based applications were executed on a specific system. With the invention of cloud computing, resources are used as an aggregated to virtual computer. This provides an environment where applications execute independently without regard for any particular configuration. 


\section{Cloud Computing Characteristics}

The essential characteristics of cloud computing can be elaborated as follows [4]:

1. On-demand self-service

2. Broad network access

3. Resource pooling

4. Rapid elasticity

5. Measured service

\section{Cloud Computing Concepts \& Benefits}

A. Below is a list of cloud computing environment attributes, describe how those attributes are realized to provide business advantages, so that the business advantages of cloud computing have been defined. With emphasis on performing the following tasks:

a. Virtualization: IT resources can be shared between many computing resources (physical servers or application servers). Provide more efficient utilization of IT resources and reduce hardware cost through resource consolidations and economies of scale. Lowering total cost of ownership and improving asset utilization.

b. Provisioning: IT resources are rapidly provisioned (or de-provisioned) based on consumer demands.Reduce IT cycle time and management cost.

c. Elastic scaling: IT environments scale up and down by any magnitudes as needed to satisfy customer demands. Optimize IT resource utilization and increase flexibility.

d. Service Automation Management: IT environments that provide the capability to request, deliver, and manage IT services automatically. Reduce IT operational costs by automating the processes used to deliver and manage a cloud computing environment.

e. Pervasiveness: Services are delivered through the use of the Internet and on any platform. Improve customers' experience by enabling services to be accessed from anywhere, at any time, and on any device.

f. Flexible pricing: Services are tracked with usage metrics to enable multiple payment models. Improve cost transparency and offer more flexible pricing schemes.

\section{Cloud Computing Building Blocks}

The cloud computing model is comprised of a front end and a back end. These two elements are connected with the help of a network or the Internet. The front end allow the user to interacts with the system; the back end is the cloud itself. The front end is composed of a client computer, or the computer network of an enterprise, and the applications used to access the cloud. The back end provides the applications, various devices, computers, servers, and data storage that creates the cloud of services.

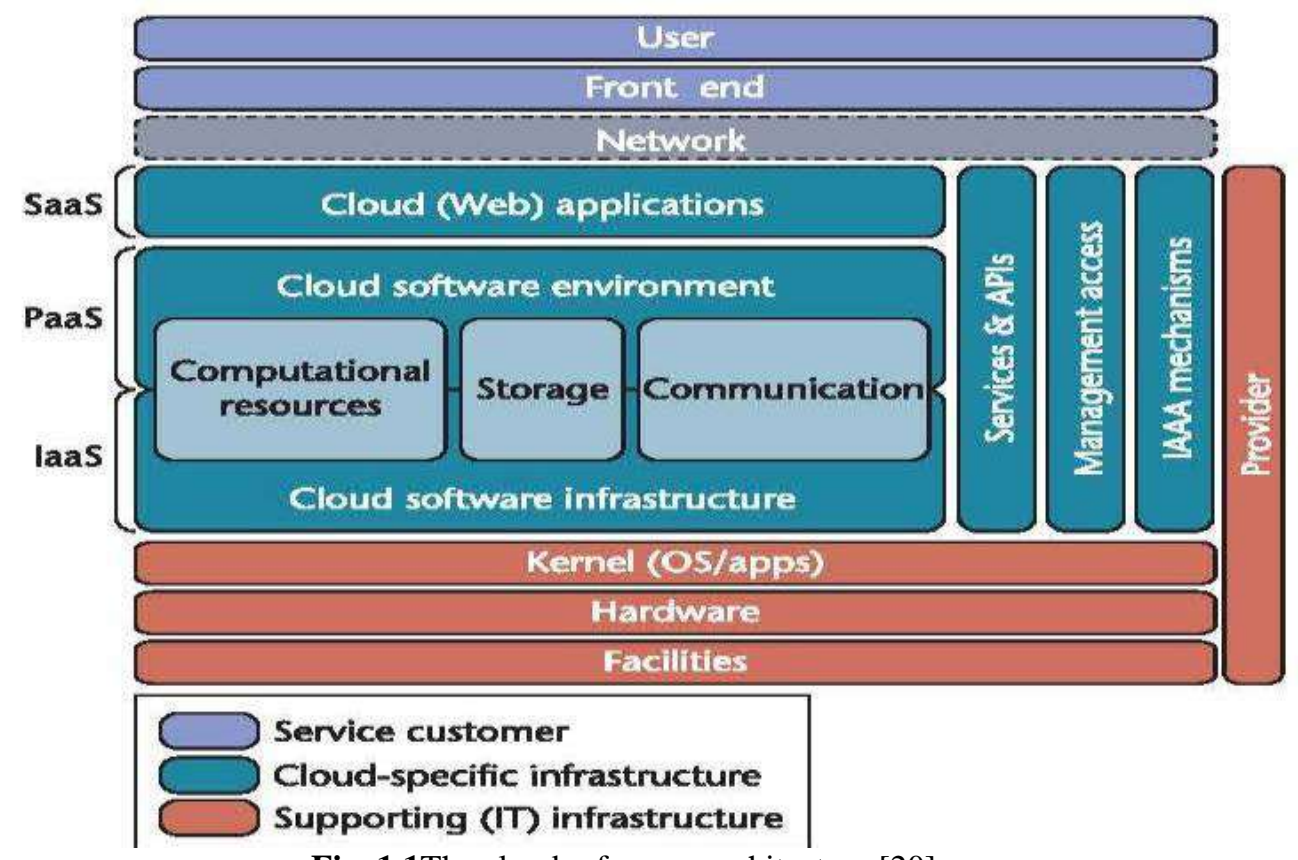

Fig. 1.1The cloud reference architecture.[20] 


\section{Virtual machine monitor (VMM)}

The virtual machine monitor (VMM) provides the means for simultaneous use of cloud facilities (see figure 1.2). VMM is a program on a host system that allows one computer to support multiple, identical execution environments. From the user's point of view, the system is a self-contained computer which is isolated from other users. In reality, every user is being served by the same machine. A virtual machine is one operating system (OS) that is being managed by an underlying control program allowing it to appear to be multiple operating systems. In cloud computing, VMM allows users to monitor and thus manage aspects of the process such as data access, data storage, encryption, addressing, topology, and workload movement.[13]

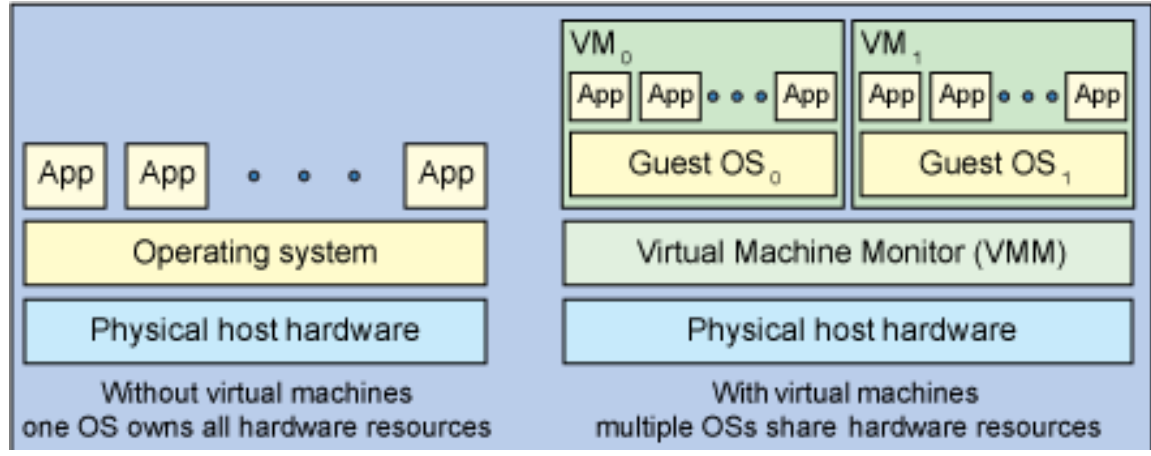

Figure 1.2 How the Virtual Machine Monitor works [13]

\section{Cluster Computing}

Cluster computing it's a group of computers connected to each other and work together as a single computer. These computers are often linked through a LAN.[8] The clusters came to existence for the high need for them, because the computing requirements are increasing in a high rate and there's more data to process, so the cluster has been used widely to improve performance. [9] The cluster is a tightly coupled system, and from its characteristics that it's a centralized job management and scheduling system. All the computers in the cluster use the same hardware and operating system, and the computers are the same physical location and connected with a very high speed connection to perform as a single computer. The resources of the cluster are managed by centralized resource manager. The cluster is single owned, to only one organization. Its interconnection network is a high-end with low latency and high bandwidth, the security in the cluster is a login/password-based, and it has a medium level of privacy depends on users privileges. It has a stable and guaranteed capacity. The self healing in the cluster is Limited, it's often restarts the failed tasks and applications its service negotiations are limited, and the user management is centralized. $[8,11]$ The cluster computing is usually used in educational resources, commercial sectors for industrial promotion \& Medical research.[2,8]

\section{Cluster Computing Characteristics}

The essential characteristics of cluster computing can be elaborated as follows [12]:

1. Tightly coupled systems

2. Single system image

3. Centralized Job management \& scheduling system

\begin{tabular}{|l|l|}
\hline Advantages & Disadvantages \\
\hline Reducing cost & Programmability Issues \\
\hline Manageability & Problem in Finding Fault \\
\hline Single System Image & Difficult to handle by a Layman \\
\hline High Availability & $\begin{array}{l}\text { Difficult for developing software for } \\
\text { distributed system }\end{array}$ \\
\hline $\begin{array}{l}\text { Improves network } \\
\text { technology }\end{array}$ & Easily accessed and applied to secret data \\
\hline
\end{tabular}

Table1-3: Advantages and Disadvantages of Cluster Computing [1,12]

\section{Grid Computing}

Grid computing is a combination of resources from multiple administrative domains to reach a common target, and this group of computers can distributed on several location and each a group of grids can be connected to each other. [2,14] The need of access to additional resources and the collaborating between organizations leads to the need for grid computing. [6] Grid environments are extremely well suited to run jobs 
that can be split into smaller chunks and run concurrently on many nodes. The grid is a loosely coupled systems and from its characteristics that it's a distributed Job Management and scheduling, the computers in the grid are not required to be in the same physical location and can be operated independently, so each computer on the grid is concerned a distinct computer, the computers in the grid are not tied to only on operating system and can run different OSs and different hardware, when it comes to a large project, the grid divides it to multiple computers to easily use their resources. The grid is multiple owned, it could be owned by several companies. Interconnection network is mostly internet with high latency and low bandwidth. The security in the grid is public and private based on authentication and mapping user to an account. And it has limited support privacy, its capacity is not stable, it varies, but it's high. The self healing in the cluster is Limited, it's often restarts the failed tasks and applications. its service negotiations are based on service level agreements, and the user management is decentralized.[2,14,15] The grid computing is usually used in predictive modeling and simulations, engineering design and automation, energy resources exploration, medical, military, basic Research and visualization. [14]

\section{Difference in Architecture Of Cloud And Grid Computing}

When viewed in a broader sense both cloud and grid computing look like one and the same thing but if we study minutely then we can see the difference between them very clearly as explained below:

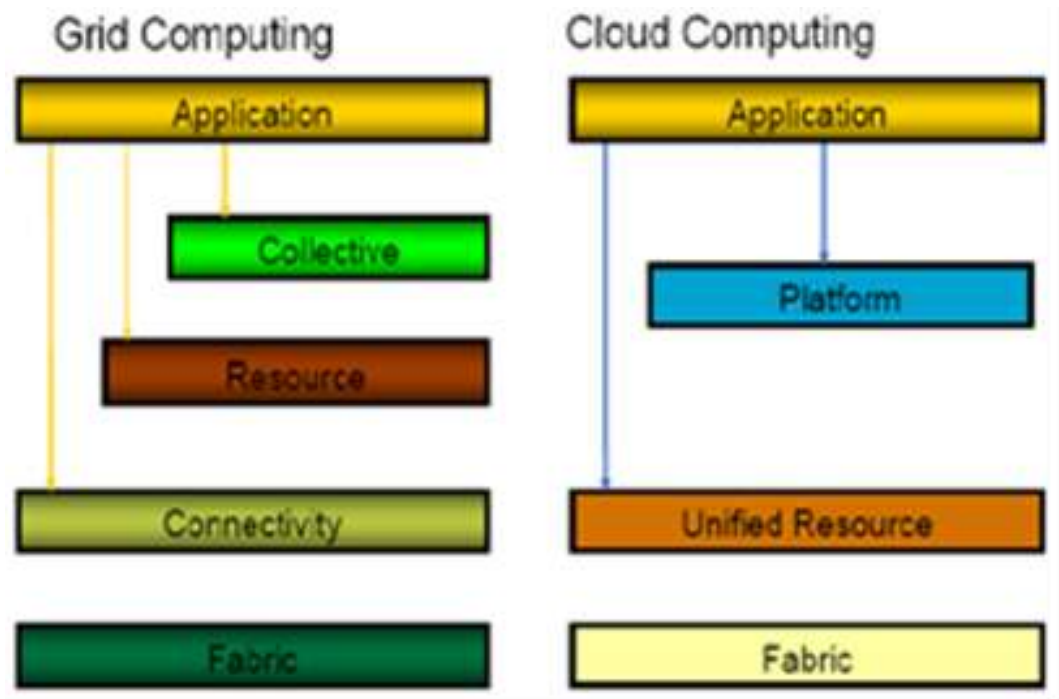

Figure 2: Grid computing and Cloud computing Architecture [21,22]

Business Models: While in grid business models are usually based on bilateral agreements between academic institutions, provision of resource in clouds requires more differentiated business models. Currently, we observe several types of business models ranging from resource providers who only provide computing resources (e. g., Amazon), over SaaS providers who sell their own resources together with their own software services (e. g., GoogleApps, Salesforce.com) to companies that attempt to run a mixed approach, i. e., they allow users to create their own services but at the same time offer their own services (Sun N1 Grid, Microsoft Azure) [24].

Resource Management: Resource management represents another major difference between grids and clouds. While grids rely on batch systems, utilization of virtualization technologies represents the resource management solution for the clouds.

Resource Provision Models: Grid resource provisioning models are based on virtual organizations where the relationships are established offline. In clouds usage of SLAs, compliance, and trust management is essential.

Resource Availability: In grids resource sharing relies on the best effort manner, sometimes resources are not available and sometimes there are plenty of resources which are idle. Clouds rely on massive elasticity in clouds. Challenging issues in clouds are to find the balance between wasting resources due to the virtualization overhead and standby modes of devices on the one hand, and pooling of resources to facilitate efficient consumption of resources and reducing energy consumption on the other. Let us differentiate two technologies on basis of features side by side. The difference between cloud and grid computing is given below in tabular form [22] [23]

\section{Utility Computing}

Utility Computing refers to a type of computing technologies and business models which provide services and computing resources to the customers, such as storage, applications and computing power.[17] This 
model has the advantage of a low cost with no initial price to reach the computer resources. This repackaging of computing services is the foundation of the shift to on demand computing, software as a service and cloud computing models which late developed the idea of computing, applications and network as a service. [17] Utility computing is kind of virtualization, that means the whole web storage space and computing power which it's available to users is much larger than the single time-sharing computer. Multiple backend web servers used to make this kind of web service possible. [18] Utility computing is similar to cloud computing and it often requires a cloud-like infrastructure. [18]

11.1 Architecture of utility computing: It depends on the devices of the users and the providing company for the utility service. And similar to the architecture of the cloud.

11.2 Advantages of utility computing: Here are some benefits of utility computing such as that the client doesn't have to buy all the hardware, software and licenses needed to do business. Instead, the client relies on another party to provide these services. It also gives companies the option to subscribe to a single service and use the same suite of software throughout the entire client organization. And it offers compatibility of all the computers in large companies. [18]

11.3 Disadvantages of utility computing: There's some issues are considered as disadvantages such as reliability which means the service could be stopped from the utility computing company for any reason such as a financial trouble or equipment problems. Also utility computing systems can also be attractive targets for hackers, and much of the responsibility of keeping the system safe falls to the provider [18]

\section{Conclusion}

In this paper, we have presented a detailed comparis on the different computing models like cluster computing, grid, utility computing and cloud computing. We believe a comparison such as this can help to understand, share and evolve infrastructure and technology within, across, and accelerate Cloud Computing from early prototypes to production systems. When it comes to grid and cloud computing, the two are often seen as the same computing paradigm under different names. In this paper, we sought to separate grids from clouds and provide a side by side comparison in how they are works, advantages disadvantages and what services are offered. In a word, the concept of cloud computing is becoming more and more popular now a days. Now cloud computing is in the beginning stage. All kinds of companies are providing all kinds of cloud computing service, from software application to net storage. We believe cloud computing will become main technology in our information life. Cloud has owned all the conditions. Now the dream of grid computing will be realized by cloud computing. It will be a great event in the IT history. Grid and cloud computing appears to be a promising model focusing on standardizing APIs, security, interoperability, new business models, and dynamic pricing systems for complex services. Hence there is a scope for further research in these areas.

\section{References}

[1]. Comparison between Cloud Computing, Grid Computing, Cluster Computing and Virtualization Rakesh Kumar Department of Information Technology JECRC, Jaipur,

[2]. Comparative study between Cluster, Grid, Utility, Cloud and Autonomic computing 1Samah Mawia Ibrahim Omer, 2Amin Babiker A.Mustafa, 3Fatema Abdallah Elmahdi Alghali

[3]. Seyyed Mohsen Hashemi, Amid Khatibi Bardsiri (MAY 2012) “Cloud Computing Vs. Grid Computing”, ARPN Journal of Systems and Software, VOL. 2, NO.5.

[4]. Siddharth Jain, Rakesh Kumar, Sourabh Kumawat, Sunil Kumar Jangir (Novenber, 2014) "An analysis of security and privacy issues, Challenges with possible solution in cloud computing", National Conference on Computational and Mathematical Sciences (COMPUTATIA-IV), Technically Sponsored By: ISITA and RAOPS, Jaipur

[5]. Rakesh Kumar, Neha Gupta, Shilpi Charu, Somya Bansal, Kusum Yadav (August, 2014) "Comparison of SQL with HiveQL", International Journal for Research in Technological Studies, Vol. 1, Issue 9, ISSN (online): 2348-1439, pg. 28-30.

[6]. Rakesh Kumar, Kanishk Jain, Hitesh Maharwal, Neha Jain, Anjali Dadhich (July, 2014) "Apache CloudStack: Open Source Infrastructure as a Service Cloud Computing Platform”, IJAETMAS, Vol.1, Issue.2, pg. 111-116, ISSN: 2349-3224.

[7]. http://edutechwiki.unige.ch/en/Grid_computing

[8]. Indu Gandotra, Pawanesh Abrol, Pooja Gupta, Rohit Uppal and Sandeep Singh - Cloud Computing Over Cluster, Grid Computing: a Comparative Analysis - Journal of Grid and Distributed Computing - Volume 1, Issue 1, 2011

[9]. Cluster, Grid, Cloud -Concepts Kalaiselvan.K - GGOA - C-DAC Bangalore - 17/03/2011

[10]. Cloud computing and emerging IT platforms: Vision, hype, and reality for delivering computing

[11]. http://utilitygridcomputing.blogspot.com/2008/10/advantages-disadvantages-of-uc.html

[12]. Siddharth Jain, Rakesh Kumar, Sourabh Kumawat, Sunil Kumar Jangir (Novenber, 2014) "An analysis of security and privacy issues, Challenges with possible solution in cloud computing", National Conference on Computational and Mathematical Sciences (COMPUTATIA-IV), Technically Sponsored By: ISITA and RAOPS, Jaipur.

[13]. Cloud computing fundamentals A different way to deliver computer resources Skill Level: Introductory Grace Walker IT Consultant Walker Automated Services.

[14]. Indu Gandotra, Pawanesh Abrol, Pooja Gupta, Rohit Uppal and Sandeep Singh - Cloud Computing Over Cluster, Grid Computing: a Comparative Analysis - Journal of Grid and Distributed Computing - Volume 1, Issue 1, 2011

[15]. http://it.toolbox.com/blogs/technews/grid-computing-advantages-and-disadvantages-23668

[16]. http://utilitygridcomputing.blogspot.com/2008/10/advantages-disadvantages-of-uc.html

[17]. http://en.wikipedia.org/wiki/Utility_computing. 
[18]. http://utilitygridcomputing.blogspot.com/2008/10/advantages-disadvantages-of-uc.html

[19]. http://gigaom.com/2008/02/28/how-cloud-utility-computing-are-different/.http://en.wikipedia.org/wiki/Utility_computing.

[20]. Cloud Computing - Research Issues, Challenges, Architecture, Platforms and Applications: A Survey Santosh Kumar and R. H. Goudar.

[21]. Comparison of Cloud and Grid Computing Sukhdev Singh Ghuman SBDSM Khalsa College, Domeli, Kapurthala, Punjab, India.

[22]. Milan Kantial Vachhani, Kishore H.Atkotiya ,"similarities and contrast between Grid Computing and Cloud Computing." Indian Journal of applied research Vol.3,Issue.3 2013.

[23]. Seyyed Mohsen Hashemi, Amid Khatibi Bardsiri," Cloud Vs. Grid computing” ARPN Journal of Systems and Software, VOL. 2, NO.5, MAY 2012.

[24]. Ivona Brandic, Schahram Dustdar, Vienna University of Technology Cloud v/s Grid Computing Technology comparison Information Technology 53 (2011) Special Issue. 